Historic, Archive Document

Do not assume content reflects current scientific knowledge, policies, or practices. 



\section{Reduction In Prices}

Having a larger supply than formerly of a num . ber of my seedling iris I have reduced the price as follows:

A. M. Brand

A. W. Blakely

Eå̀ch 13 Pér 10

A. E. Kunderd.

$\$ 3.00$

Aunt Rachel

Barton Harrington

Camelia

$\begin{array}{lll}40 & 40 & \$ 0\end{array}$

C. D. Hayes

80

$60 \ldots-5.00$

5.00

.80

Clarence Wedge

Cora Vernon.

9.00

Dr. J. W. Andrist

Dr. A. F. Mantor

1.00

1.00

3.00

5.00

.80

E. C. Shaw.

7.00

Fryer's Glory

10.00

Grace E. Kimball

4.00

G. W. Peake

1.25

Jessie McInnis

1.00

Lowell (No. 32)

80

.75

7.00

Mrs. A. M. Brand

1.00

6.50

Mrs. K. Andrist

Mrs. Bossart

2.50

Mrs. A. M. Brand.

5.00

Miss Bessie (No. 31)

1.00

Mrs. J. S. Brand.

1.00

Madison Cooper

3.50

Mrs. W. F. Christman

5.00

Mrs. C. D. Hayes

4.00

2.50

Mrs. W. F. Kinball

Minnesota

3.50

M. G. Peters

2.50

Margaret Sheridan.

3.50

Mort W. Sanford

Mrs. M. W. Sanford

Mrs. S. H. Smith

1.00

2.00

Red Glory.

5.00

.40

1.00

Rev. S. H. Smith

1.75

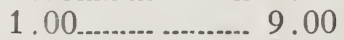

Rev. A. H. Wurtelle

W. F. Christman.

Mixed seedlings of tall bearded iris $\$ 6.00$ per 100 , 5 at 10 rates.

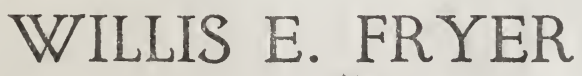

MANTORVILLE, MINN., U. S. A.

Sent postpaid in the U.S. 
THIS IS THF ONLY CATALOG I HAVF AT FRESENT.

TE.FRYER • 